\title{
ÚLTIMAS PALABRAS EN EL CORREDOR DE LA MUERTE: UN ANÁLISIS FENOMENOLÓGICOđ
}

\author{
Álvaro Málaga ${ }^{(}$, \& Ana R. Delgado ${ }^{1}(\mathbb{D}$ \\ Universidad de Salamanca, Salamanca, España. ${ }^{2}$
}

\section{RESUMEN}

Este artículo presenta un análisis fenomenológico cuyo objetivo es conocer la experiencia de los condenados a muerte a partir de sus últimas palabras. La muestra se compone de 61 transcripciones de las personas ejecutadas por el Estado de Texas entre 2011 y 2017, que fueron analizadas temáticamente. Entre los temas principales se encuentran la expresión de afecto, las creencias religiosas, el arrepentimiento, la aceptación, el agradecimiento y el sentido de cierre. La mayor parte de los temas hallados son de carácter positivo, lo que es coherente con los hallazgos de investigaciones previas. Al ser un método experiencial y descriptivo, la evaluación de la integridad metodológica ofrece escasa dificultad.

\section{Palabras Claves}

corredor de la muerte; fenomenología descriptiva; fidelidad; integridad metodológica; utilidad

\begin{abstract}
This paper describes a phenomenological analysis whose goal is to learn the experience of death row inmates from their last statements. The sample is composed of 61 transcriptions from people executed by the U.S. state of Texas between 2011 and 2017, that were analyzed thematically. Among the main themes we found affection, religious belief, regret, acceptance, gratefulness and sense of closure. Most themes were of positive valence, which is coherent with previous research results. Because we used an experiential and descriptive method, the assessment of methodological integrity is uncomplicated.
\end{abstract}

\section{Keywords}

death row; descriptive phenomenology; fidelity; methodological integrity; utility

\footnotetext{
${ }^{1}$ Correspondence about this article should be addressed to Ana R. Delgado: adelgado@usal.es

${ }^{2}$ Facultad de Psicología, Avda. de la Merced 109-131, 37005 Salamanca, España.
} 


\section{DEATH ROW LAST STATEMENTS: A PHENOMENOLOGICAL ANALYSIS}

\section{Introducción}

En el ámbito criminológico, la restricción del acceso directo de los académicos a las instituciones penales (Simon, 2000) ha llevado a los investigadores a plantear alternativas innovadoras en sus proyectos. Así, las investigaciones sobre el castigo y la pena capital utilizan cada vez con más frecuencia estrategias de recogida de datos basadas en artefactos sociales, tales como transcripciones de intervenciones habladas, obras de ficción o documentos oficiales (Lynch, 2015). Las estrategias disponibles para el análisis de los datos cualitativos así obtenidos son también múltiples, aunque, en último término, los procedimientos empleados no sean muy diferentes. Por ejemplo, el proyecto metodológico de Wertz et al. (2011), comparó los resultados del análisis de una misma entrevista mediante varios métodos, como el fenomenológico, el de la teoría enraizada (ambos clasificados como temáticos por Pistrang \& Barker, 2012), el narrativo, centrado en cómo el relato da sentido a la experiencia, y el análisis del discurso, que estudia la interacción social vía lenguaje, y concluyó que se daban elementos procedimentales comunes, algunos de los cuales pueden verse en la Tabla 1.

\section{Tabla 1}

Algunos elementos procedimentales comunes a distintos tipos de análisis.

Se parte de descripciones o expresiones contextualizadas.

Los resultados se exponen por medio del lenguaje descriptivo ordinario.

Se atiende a los aspectos corpóreos, emocionales, sociales, culturales o temporales.

Se seleccionan partes del texto relevantes para los objetivos del investigador.

Se identifican patrones recurrentes en los datos, el diseño es emergente.

Control mediante el retorno a los datos.

Fundamentación del conocimiento en la evidencia.

Transparencia en los procedimientos.

Reflexividad, actitud crítica en relación con los límites del análisis y sus resultados.

Ha habido otras propuestas sobre lo que es común a toda investigación cualitativa. La afirmación de Rennie (2012) de que todos los métodos son hermenéuticos o interpretativos fue rápidamente rebatida por Giorgi (2014), cuya propuesta metodológica es descriptiva, en el sentido que damos a este término en el lenguaje ordinario. Una diferencia clave en este contexto es la que se da entre enfoques experienciales y discursivos (Reicher, 2000). Los enfoques experienciales son más conservadores epistemológicamente, pues asumen que el lenguaje da acceso a la realidad mental (aunque imperfectamente, según los supuestos del realismo crítico o postpositivismo característico de la ciencia normal en estos momentos); los discursivos no, pues conceptualizan el lenguaje como una forma de acción social que sirve para construir nuestro mundo (Berger 
\& Luckmann, 1966). Un análisis temático, por ejemplo, podría hacerse desde cualquiera de esas perspectivas, por lo que es imprescindible diferenciar procedimiento y enfoque.

En lo que respecta a los criterios de calidad de las investigaciones cualitativas, la integridad metodológica se evalúa a partir de la fidelidad a la materia objeto de estudio y la utilidad con respecto a la consecución de los objetivos de la investigación (Levitt et al., 2018). ¿Se han seleccionado procedimientos coherentes con el fenómeno que se estudia tal y como se concibe desde el enfoque empleado? En este caso, la fidelidad mejora, como también lo hace si se recogen datos adecuados, de fuentes que pueden arrojar luz sobre las variaciones del fenómeno que son relevantes para el objetivo del estudio; si hay un adecuado manejo de la perspectiva en la recogida de los datos, es decir, se reconoce reflexivamente (Gough \& Madill, 2012) la influencia del punto de vista y se limita, tratando de ser transparentes; si hay un adecuado manejo de la perspectiva en el análisis de los datos; y, finalmente, cuando los resultados están enraizados, es decir, hay una fundamentación del conocimiento en la evidencia, en los datos (que otros podrían auditar, en ciertos procedimientos).

La utilidad para la obtención de los objetivos de la investigación se ve reforzada cuando se contextualizan los datos, se seleccionan los que ofrecen mayor potencial para el insight teórico, dando lugar a contribuciones significativas, y cuando hay coherencia entre diferentes resultados. La valoración ha de hacerse siempre considerando el diseño de la investigación en conjunto, es decir, teniendo en cuenta (1) los objetivos (p.ej., describir la experiencia subjetiva), (2) el enfoque (p.ej., fenomenológico) y (3) las características metodológicas (p.ej., análisis temático, en un lugar, con unos participantes y unos investigadores concretos). La confianza en los hallazgos de una investigación cualitativa aumenta en la medida en que los procedimientos se emplean de forma coherente con el diseño.

En nuestra investigación, el objetivo era conocer el significado que dan los presos del corredor de la muerte al hecho de enfrentarse a su propia ejecución, por lo que el enfoque fenomenológico es el más recomendable. En cuanto al análisis, el adecuado sería el temático, ya que se trata de describir las ideas principales identificadas en los datos (Braun \& Clarke, 2012; Pistrang \& Barker, 2012). En conjunto, se trataría del método fenomenológico-descriptivo, cuyo perfil de metodología especial no ofrece lugar a dudas (Delgado, 2010, 2013; Giorgi, 2014; Giorgi \& Giorgi, 2003). 


\section{Últimas palabras de los condenados a muerte}

El Estado de Texas hace públicas en su página web las trancripciones de las últimas palabras de todos los presos ejecutados desde diciembre del 1982. En el momento de la ejecución pueden estar presentes sus familiares directos, a quienes se da la oportunidad de invitar a amigos y abogados implicados en el proceso de defensa. Desde 1996, a petición de las asociaciones de víctimas, también pueden estar presentes los familiares de las víctimas de los delitos cometidos por el condenado (Texas Department of Criminal Justice, 2017).

Existen algunas investigaciones sobre transcripciones previas, como la de Heflick (2005), que estudió cualitativamente las últimas palabras de 237 condenados entre enero de 1997 y abril de 2005, hallando seis temas: creencias relativas a lo que hay después de la muerte (especialmente el cielo cristiano), activismo con temática social, muestras de afecto, declaración de inocencia, búsqueda de perdón y negativa a hacer una declaración. Shuck y Ward (2008) analizaron 283 textos recogidos entre diciembre de 1982 y noviembre de 2006; aunque su objetivo era analizar el discurso (y, por tanto, el foco estaba en la interacción social y no en la experiencia subjetiva), crearon previamente un marco temático y hallaron reflejados, de mayor a menor frecuencia, los siguientes contenidos: expresar amor, dirigirse a otros, religión, búsqueda de perdón, confort, deseos, declaración de culpabilidad, declaración de inocencia, activismo político, amenazas, descripciones de atributos personales, circunstancias relacionadas con el crimen y no estar en paz.

Rice, Dirks y Exline (2009) estudiaron 345 transcripciones, de diciembre de 1982 a junio de 2005, con objeto de comparar las últimas palabras de los presos antes y después del 12 de enero de 1996, momento en el que se empezó a permitir a los allegados a la víctima acudir a la ejecución de los presos; encontraron que, a partir de esa fecha, eran menos los reclusos que se negaban a hacer una última declaración y más los que admitían ser culpables, dando muestras de arrepentimiento.

Otros estudios han empleado técnicas cualitativas, partiendo de códigos preestablecidos, para analizar las últimas palabras de los condenados del corredor de la muerte de Texas, encontrando que el tema más común es el amor, seguido de la espiritualidad; el arrepentimiento y la solicitud de perdón son también usuales. Sus análisis terminan con las transcripciones del 31 de julio de 2011 (Foley \& Kelly, 2007; Kelly \& Foley, 2013). La fecha es relevante aquí, ya que nuestro análisis, cuyo objetivo 
es conocer la experiencia subjetiva de los condenados del corredor de la muerte antes de ser ejecutados, comienza con las transcripciones del día siguiente.

\section{Método}

Se ha empleado el método fenomenológico-descriptivo, es decir, un análisis de tipo temático y enfoque fenomenológico.

\section{Muestra}

Analizamos las transcripciones de las últimas palabras pronunciadas por los 70 presos ejecutados por el Estado de Texas entre el 1 de agosto de 2011 y el 31 de mayo de 2017; son de dominio público y pueden hallarse en la página del Departamento de Justicia Criminal, http://tdcj.state.tx.us/death_row/dr_executed_offenders.html. De los 70 prisioneros, 9 declinaron hacer declaraciones o simplemente permanecieron en silencio, por lo que el análisis se ha llevado a cabo sobre 61 textos.

\section{Procedimiento}

Siguiendo el método fenomenológico-descriptivo, se llevó a cabo el análisis en cuatro pasos: (1) Lectura de todas las transcripciones, para tener una noción del sentido completo del mismo. (2) Determinación de las partes para establecer unidades de significado: se relee cada transcripción con una actitud fenomenológica (o reflexiva, en términos más abstractos, tratando de limitar cualquier carga teórica relacionada con el fenómeno a estudiar) y se van señalando los cambios de significado en el lenguaje ordinario. (3) Transformación de las unidades de significado en expresiones psicológicamente sensibles: proceso de refinamiento progresivo en el que las unidades de significado establecidas en un primer momento se van modificando para acercarse a un vocabulario psicológico con respecto al fenómeno estudiado. Es usual emplear tablas para ilustrar los temas con ejemplos seleccionados de las transcripciones. (4) Determinación de la estructura (los fenomenólogos tradicionales de orientación filosófica hablarían de esencia) a partir de los significados más comunes y/o conceptualmente relevantes conectados a la experiencia, lo que permite aumentar el insight teórico, uno de los criterios de utilidad. 


\section{Resultados}

La Tabla 2 muestra los temas que reflejan la experiencia común de los presos antes de ser ejecutados con ejemplos de unidades de significado.

Los temas que se encuentran en las transcripciones de un mayor número de casos son, en orden decreciente: (1) expresión de afecto, que suele estar dirigido a familiares o amigos, aunque otras veces se describe en términos generales o se refiere a los compañeros del corredor o al personal de la cárcel; (2) creencias religiosas, en general procedentes del cristianismo, tales como Dios, Jesucristo o el cielo; (3) agradecimiento, que de manera semejante a expresión de afecto suele ir dirigido a familiares y amigos, pero también a abogados, personal de la cárcel, o hallarse en términos genéricos; (4) aceptación de la muerte, dicen estar preparados para ese momento; (5) arrepentimiento, dirigido sobre todo a los familiares y allegados de la víctima; (6) infundir ánimos a las personas que se encuentran en la sala, generalmente sin especificar a quién, pidiendo que se mantenga la entereza ante la inminente ejecución; (7) sentido de cierre, expresando el deseo de que su muerte sirva para que se ponga fin a una situación difícil, especialmente para los familiares de las víctimas; (8) empatía, el preso se pone en lugar de otras personas; (9) bienestar, expresando que se encuentran en un momento de paz; (10) situación percibida como injusta, en la que el ejecutado protesta contra la moralidad del acto o contra la verdad de las acusaciones; (11) búsqueda de perdón, petición expresa por parte del preso de que se le perdone por los actos cometidos.

El tema empatía puede subdividirse en función de las personas a las que se dirige el preso. En cualquier caso, se habla de una "empatía cognitiva”, en el sentido de que el condenado se pone en el lugar de la otra persona, pero no está manifestando tener los mismos sentimientos que ella. Encontramos que se expresa especialmente en relación con los familiares de las víctimas, seguida de la empatía con sus compañeros del corredor y con sus propios familiares. Semejante es el caso del tema deseos positivos: se expresan mayoritariamente deseos positivos hacia los familiares de las víctimas, pero también hacia sus propios familiares y hacia los compañeros del corredor de la muerte.

Puede verse que la mayoría de los temas son de valencia positiva. Los casos en los que se expresan sentimientos negativos son menos, y suelen estar relacionados con el arrepentimiento por el crimen cometido; los insultos, la rabia o el odio son anecdóticos o simplemente inexistentes: los condenados, en general, dejan un mensaje positivo antes de morir. 
Tabla 2

Temas que aparecen al menos en cinco casos y ejemplo.

\begin{tabular}{|c|c|}
\hline Tema & Ejemplo \\
\hline Expresión de afecto & "Os quiero a todos." \\
\hline Creencias religiosas & "Que todos vayamos al cielo." \\
\hline Agradecimiento & "Gracias por estar aqui."” \\
\hline Aceptación & “Estoy preparado." \\
\hline Arrepentimiento & "Siento lo que he hecho." \\
\hline Infundir ánimos & "Sed fuertes por mi. Mantened la cabeza alta." \\
\hline Sentido de cierre & $\begin{array}{l}\text { "Espero que esto os permita cerrar el asunto y hallar respuestas a } \\
\text { algunas preguntas." }\end{array}$ \\
\hline Empatía* & $\begin{array}{l}\text { "Puedo comprender vuestra indignación y por qué estáis enfadados } \\
\text { conmigo." }\end{array}$ \\
\hline Bienestar & "Estoy bien, estoy OK." \\
\hline $\begin{array}{l}\text { Situación percibida como } \\
\text { injusta }\end{array}$ & "Se están dando un montón de injusticias con esto. Esto es un error." \\
\hline Búsqueda de perdón & "Por favor, perdonadme." \\
\hline Recado & "Mamá, cuida de mi hija." \\
\hline Deseos positivos* & $\begin{array}{l}\text { "Espero que puedan encontrar la paz y ser productivos para la } \\
\text { sociedad." }\end{array}$ \\
\hline Declaración de inocencia & "Mamá, Celeste: por favor, sabed que soy inocente." \\
\hline Resignación & "Estoy dispuesto." \\
\hline $\begin{array}{l}\text { Que las víctimas no } \\
\text { guarden rencor }\end{array}$ & "Por favor, no guarden rencor. Es lo que quiero." \\
\hline Consejo vital & $\begin{array}{l}\text { "Jóvenes, escuchad a vuestros padres; haced siempre lo que os digan, id } \\
\text { a la escuela, aprended de vuestros errores." }\end{array}$ \\
\hline Despedida & "Adiós." \\
\hline Hablar sobre el caso & "El único testigo de lo que ocurrió aquella noche es Dios." \\
\hline Búsqueda de la verdad & "Pediría que se sepa la verdad y se hagan cambios positivos." \\
\hline $\begin{array}{l}\text { Manifestar cambio como } \\
\text { persona }\end{array}$ & "Vine como un león y me voy tan pacífico como un cordero." \\
\hline
\end{tabular}

A la estructura de la experiencia se llega conectando los temas, lo que permite facilitar el insight teórico, uno de los criterios de utilidad. Los vínculos más estrechos entre los temas experienciales se establecen entre creencias religiosas y bienestar; expresión de afecto, agradecimiento e infundir ánimos y finalmente entre arrepentimiento, sentido de cierre y búsqueda de perdón. Un tema muy común, situación percibida como injusta está conceptualmente vinculado a declaración de inocencia. Esto podría llevar a una indagación posterior en términos del receptor del mensaje: por un lado hay parte del contenido que está centrado en el propio preso, una segunda parte del contenido que está dirigido a los seres queridos del ejecutado, a los que quieren mostrar amor y agradecimiento, y una tercera parte dirigida a los familiares de las víctimas, ante los cuales se muestran arrepentidos y piden perdón, o desean que su propia ejecución les sirva para poner cierre a esa desagradable situación. Sin embargo, en relación con la fidelidad a la materia objeto de estudio, que es en este caso la experiencia, el manejo de la perspectiva en el análisis de datos nos permite simular mentalmente distintos focos para el análisis y defender que es más adecuado un análisis de tipo fenomenológico 
descriptivo que un análisis del discurso, centrado en la interacción simbólica con los presentes en la sala, receptores del discurso, que sí podría ser el indicado cuando el estudio se lleva a cabo con objetivos de tipo sociológico o legal.

\section{Discusión}

Los resultados del análisis fenomenológico son coherentes con investigaciones previas como las de Heflick (2005): aunque la etiqueta verbal de los temas no sea idéntica, la expresión de afecto hacia los seres queridos en los momentos previos a la ejecución y las creencias religiosas aparecen en un gran número de casos. Además, en ambas investigaciones se hallan temas relacionados con la situación percibida como injusta (activismo con temática social en Heflick, 2005) e infundir ánimos (activismo centrado en la preocupación de aquellos que deja atrás en Heflick, 2005). También coinciden en la importancia de la búsqueda de perdón y la declaración de inocencia.

Aunque nuestro enfoque es fenomenológico, experiencial, y el de Shuck y Ward (2008) es discursivo, hay coherencia en los resultados relativos a los contenidos más relevantes: la expresión de sentimientos, sobre todo positivos, en las palabras de los condenados, así como la aceptación o el rechazo de la situación en los temas aceptación y situación percibida como injusta respectivamente.

En cuanto a los resultados de investigaciones llevadas a cabo sobre las transcripciones anteriores (Foley \& Kelly, 2007; Kelly \& Foley, 2013) a las aquí analizadas, los dos temas más importantes fueron amor (en nuestro estudio: expresión de afecto) y espiritualidad (en nuestro estudio: creencias religiosas).

Junto a la coherencia con las conclusiones de investigaciones previas, es de resaltar la detección de dos nuevos temas, agradecimiento y sentido de cierre. Esto indicaría que la saturación no había sido alcanzada en análisis temáticos previos. Finalmente, se aporta una visión de conjunto de la experiencia subjetiva a partir de las relaciones entre los temas más relevantes. La estructura se ha descrito verbalmente, aunque empieza a ser usual emplear recursos gráficos, especialmente cuando el objetivo es la construcción de teoría enraizada, denominación de uno de los métodos cualitativos mejor articulados, aunque epistemológicamente sea menos consistente que el método fenomenológico-descriptivo. Al ser este último un método experiencial y descriptivo, la evaluación de la integridad metodológica (fidelidad a la materia objeto de estudio y utilidad con respecto a la consecución de los objetivos) ofrece escasa dificultad. 
En relación con la experiencia descrita, sería de especial interés contar con estudios similares en culturas diferentes a la de los Estados Unidos. En la página web de Amnistía internacional (2018) puede verse un mapa interactivo donde se identifican países como China, Irán o Arabia Saudí en cuya legislación se mantiene la pena de muerte, pese a la evidencia en contra de su efectividad como medida disuasoria (p.ej. Death Penalty Information Center, 2018). No hay razones convincentes para sospechar que las últimas palabras de los condenados tengan una gran carga de deseabilidad social, por lo que los abolicionistas podrían contar con un argumento añadido en la evidencia de rehabilitación que se desprende de las últimas palabras de la mayoría de los presos del corredor de la muerte de Texas.

No deja de ser curioso que una de las citas más difundidas sobre el concepto de realidad -lo que no desaparece, aunque dejes de creer en ello- proceda de un relato de ciencia-ficción (Espero llegar pronto, publicado en 1980 por Philip K. Dick). Los métodos cualitativos que estudian la experiencia subjetiva de los otros, es decir, los de orientación fenomenológica, cobran sentido porque asumimos que esa realidad existe y que es posible conocerla, aunque solo sea imperfecta y gradualmente. Estos son los supuestos del realismo crítico, los que sustentan la ciencia psicológica (Delgado, 2010; Michell, 2004). Existen otros métodos cualitativos, algunos de ellos muy empleados por los clínicos, que rechazan la herencia metodológica del conductismo y del experimentalismo cognitivista (Braun \& Clarke, 2013). Habrá que atender a su evolución en los próximos años, pues no hay motivo para menospreciar a priori la aportación de formas de conocimiento que son, de hecho, empleadas por los científicos, aunque sus procedimientos estén, a menudo, ausentes de los informes de investigación (Delgado \& Prieto, 1997). 


\section{Referencias}

Amnesty International (2018, March 21). The global view. Death sentences and executions 2007-2016. Retrieved from https://www.amnesty.org/en/whatwedo/death-penalty/

Berger, P. L., \& Luckmann, T. (1966). The social construction of reality. Garden City, NY: Doubleday \& Co., Inc.

Braun, V. \& Clarke, V. (2012). Thematic analysis. En H. Cooper, P. M. Camic, D. L. Long, A. T. Panter, D. Rindskopf, \& K. J. Sher (Eds), APA handbook of research methods in psychology, Vol. 2: Research designs: Quantitative, qualitative, neuropsychological, and biological (pp. 57-71). Washington, DC: American Psychological Association. https://doi.org/10.1037/13620-004

Braun, V. \& Clarke, V. (2013). Successful qualitative research: A practical guide for beginners. London: Sage.

Death Penalty Information Center (2018, March 21). Deterrence: States Without the Death Penalty Have Had Consistently Lower Murder Rates. Retrieved from https://deathpenaltyinfo.org/deterrence-states-without-death-penalty-have-hadconsistently-lower-murder-rates

Delgado, A. R. (2010). Metodología especial, métodos cualitativos y conceptos abstractos. Psicothema, 22, 509-512.

Delgado, A.R. (2013). Why include phenomenological analysis in a Research Methods course? Psicothema, 25, 227-231. https://doi.org/10.7334/psicothema2012.180

Delgado, A.R., \& Prieto, G. (1997). Introducción a los métodos de investigación de la psicología. Madrid: Pirámide.

Foley, S. R., \& Kelly, B. D. (2007). The psychological concomitants of capital punishment: Thematic analysis of last statements from death row. American Journal of Forensic Psychiatry, 28, 7-14.

Giorgi, A. (2014). An affirmation of the phenomenological psychological descriptive method: A response to Rennie (2012). Psychological Methods, 19, 542-551. https://doi.org/10.1037/met0000015

Giorgi, A., \& Giorgi, B.M. (2003). The descriptive phenomenological psychological method. In P. M. Camic, J. E. Rodes, \& L. Yardley (Eds.), Qualitative research in Psychology. Expanding perspectives in methodology and design (pp. 243-273). Washington, D. C: APA.

Gough, B., \& Madill, A. (2012). Subjectivity in psychological science: from problem to prospect. Psychological Methods, 17, 374-384. https://doi.org/10.1037/a0029313

Heflick, N. A. (2005). Sentenced to die: last statements and dying on death row. Omega, 51, 323-336. https://doi.org/10.2190/96X8-FLUT-TCLH-EL71

Kelly, B. D., \& Foley, S. R. (2013). Love, spirituality, and regret: Thematic analysis of last statements from death row, Texas (2006-2011). The Journal of the American Academy of Psychiatry and the Law, 41, 540-550.

Levitt, H. M., Bamberg, M., Creswell, J. W., Frost, D. M., Josselson, R., \& SuárezOrozco, C. (2018). Journal article reporting standards for qualitative primary, qualitative meta-analytic, and mixed methods research in psychology: The APA Publications and Communications Board task force report. American Psychologist, 73, 26-46. https://doi.org/10.1037/amp0000151

Lynch, M. (2015). Penal artifacts: mining documents to advance punishment and society theory. En J. Miller, \& W. Palacios (Eds.), Qualitative Research in Criminology (pp. 271-290). London, UK: Transaction Publishers. 
Madill, A., \& Gough, B. (2008). Qualitative research and its place in psychological science. Psychological Methods, 13, 254-271. https://doi.org/10.1037/a0013220

Michell, J. (2004). The place of qualitative research in psychology. Qualitative Research in Psychology, 1, 307-309. https://doi.org/10.1191/1478088704qp020oa

Pistrang, N. \& Barker, C. (2012). Varieties of qualitative research: a pragmatic approach to selecting methods. En H. Cooper, P. M. Camic, D. L. Long, A. T. Panter, D. Rindskopf, \& K. J. Sher (Eds), APA handbook of research methods in psychology, Vol. 2: Research designs: Quantitative, qualitative, neuropsychological, and biological (pp. 5-18). Washington, DC: American Psychological Association https://doi.org/10.1037/13620-001

Poortman, C.L., \& Schildkamp, K. (2012). Alternative quality standards in qualitative research? Quality and Quantity, 46, 1727-1751. https://doi.org/10.1007/s11135011-9555-5

Reicher, S. (2000). Against methodolatry. Some comments on Elliott, Fischer, and Rennie. British Journal of Clinical Psychology, 39, 1-6. https://doi.org/10.1348/014466500163031

Rennie, D. L. (2012). Qualitative research as methodical hermeneutics. Psychological Methods, 17, 385-398. https://doi.org/10.1037/a0029250

Rice, S. K., Dirks, D., \& Exline, J. J. (2009). Of guilt, defiance, and repentance: Evidence from the Texas death chamber. Justice Quarterly, 26, 295-326. https://doi.org/10.1080/07418820802178063

Schuck, A. R. T., \& Ward, J. (2008). Dealing with the inevitable: Strategies of selfpresentation and meaning construction in the final statements of inmates on texas death row. Discourse and Society, 19, 43-62. https://doi.org/10.1177/0957926507083687

Simon, J. (2000). The "society of captives" in the era of hyper-incarceration. Theoretical Criminology, 4, 285-308. https://doi.org/10.1177/1362480600004003003

Texas Department of Criminal Justice. (2017). Victim survivors viewing executions: Frequently asked questions. Recuperado el 1 de marzo, 2018, de http://www.tdcj.texas.gov/faq/victim_viewing_execution.html

Wertz, F. J., Charmaz, K., McMullen, L. M., Josselson, R., Anderson R., \& McSpadden E. (2011). Five ways of doing qualitative analysis: Phenomenological psychology, grounded theory, discourse analysis, narrative research, and intuitive inquiry. New York: The Guilford Press.

Received: 2018/07/06 Accepted: 2020/02/12 